
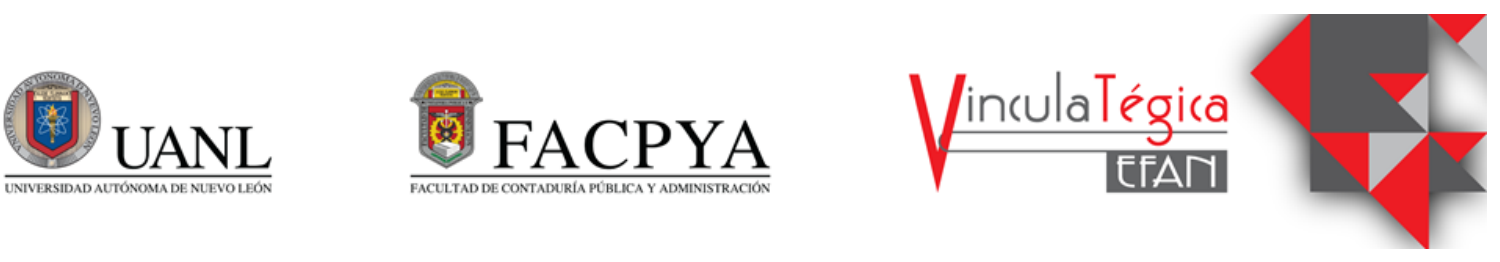

\title{
Uso del smartphone entre jóvenes universitarios en tiempos de pandemia
}

\author{
Mayra Elizabeth Brosig Rodríguez ${ }^{1}$, Claudia Ivonne Niño Rodríguez ${ }^{2}$ y Jesús Cantú \\ Rodríguez ${ }^{3}$
}

\author{
${ }^{1}$ Universidad Autónoma de Nuevo León, Facultad de Contaduría Pública y Administración \\ Monterrey, Nuevo León, México, dra.elizabethbrosig@hotmail.com, Av. Universidad S/N Col. \\ Ciudad Universitaria, San Nicolás de los Garza, Nuevo León 8129265101 \\ ${ }^{2}$ Universidad Autónoma de Nuevo León, Facultad de Contaduría Pública y Administración \\ Monterrey, Nuevo León, México, clau_nino@live.com.mxAv.Universidad S/N Col.Ciudad \\ Universitaria, San Nicolás de los Garza, Nuevo León. 8118646888 \\ ${ }^{3}$ Universidad Autónoma de Nuevo León, Facultad de Contaduría Pública y Administración \\ Monterrey, Nuevo León, México, jesus.canturo@uanl.mxAv.Universidad S/N Col. Ciudad Universitaria, San \\ Nicolás de los Garza, Nuevo León 8180202129
}

Información del artículo revisado por pares

Fecha de aceptación: junio-2021

Fecha de publicación en línea: diciembre-2021

DOI: https://doi.org/10.29105/vtga7.1-161

\begin{abstract}
En estos tiempos de pandemia la educación tiene nuevos escenarios de aprendizaje, en los cuales los estudiantes y maestros se han visto en la necesidad de actualizar la práctica educativa, mediante el uso y aplicación de herramientas digitales, que les permite trascender a una era virtual de conectividad total; con llevando toda esta actualización al progreso tecnológico del cual serán parte y permitirá grandes beneficios a favor de la educación.

La presente investigación tiene como propósito identificar el uso del smartphone en tiempos de pandemia en los estudiantes de séptimo semestre de la carrera de Licenciados en Administración, para determinar beneficios y dificultades como herramienta de apoyo en el proceso de enseñanzaaprendizaje y evaluación de estudiantes universitarios. Se realiza un análisis factorial del bloque 2 del cuestionario "Smartphone y Universidad. Visión del alumnado" medición que comprende la relación académica con el smartphone, a través de este análisis de datos se muestran como resultados el uso masivo y
\end{abstract}

prolongado del smartphone para actividades académicas, además de reconocer como beneficios su utilidad y acceso a la información, y como dificultades las interrupciones en las sesiones y el desvío de atención durante el desarrollo del proceso educativo.

Palabras clave: Innovación, innovación tecnológica, innovación social.

Clasificación JEL: O310, O350.

\begin{abstract}
In these times of pandemic, education has new learning scenarios, in which students and teachers have seen the need to update educational practice, through the use and application of digital tools, which allows them to transcend a virtual era of full connectivity; with bringing all this update to technological progress of which they will be part and will allow great benefits in favor of education.

The purpose of this research is to identify the use of the Smartphone in times of pandemic in students of the seventh semester in the LA career, to
\end{abstract}


determine benefits and difficulties as a support tool in the teaching-learning process and evaluation of university students. A factorial analysis of block 2 of the questionnaire "Smartphone and University. Vision of the students" measurement that includes the academic relationship with the Smartphone, through this data analysis the massive and prolonged use of the smartphone for academic activities is shown as results, in addition to recognizing its usefulness and access to information as benefits, and as difficulties the

\section{INTRODUCCIÓN}

El mundo está cambiando y la pandemia de COVID 19 nos ha obligado a implementar cambios significativos en la manera de vivir y llevar nuestra vida diaria; y en la educación no es la excepción, los ajustes realizados en el proceso de enseñanza - aprendizaje vinieron a agilizar la innovación educativa necesaria en esta globalización.

Sin embargo, al hablar de innovación educativa, no solo se refiere al uso de las tecnologías de información y comunicación, implica realizar esfuerzos de todos los involucrados, instituciones, estudiantes y docentes en renovarse, mostrar apertura a los cambios y actualizaciones, además de compromiso en los procedimientos de enseñar y aprender.

Si la intención es educar es preparar para la vida, los docentes en su rol de facilitadores en estos tiempos tecnológicos deben abordar su práctica educativa hacia el estudiante adaptada al uso y empleo de las nuevas tecnologías, de tal manera que los envuelva en el interés de explorar lo nuevo, al permitir pensar y aprender de manera interactiva, considerando que las generaciones universitarias actuales son expansivas en el uso del internet y por lo tanto en el uso de dispositivos móviles tales como teléfonos inteligentes, tabletas, asistentes digitales personales, etc.

Podemos definir teléfono inteligente o smartphone, como un dispositivo móvil en el que se pueden tener funciones de teléfono celular con mayor conectividad, computadora $\mathrm{u}$ ordenador de bolsillo, en el cual se pueden realizar multitareas, funciones multimedia interruptions in the sessions and the diversion of attention during the development of the educational process.

Keywords: Innovation, technological innovation, social innovation.

\section{JEL classification: O310, O350}

(cámara y reproductor de audio/video) con acceso al correo electrónico, internet vía wifi o redes, además de programas de navegación, así como el poder leer documentos en formatos como pdf, html, txt. Además de tener la opción de instalar aplicaciones adicionales según el gusto y necesidad del usuario.

El propósito de esta investigación es identificar el uso del Smartphone en tiempos de pandemia en los estudiantes de séptimo semestre en la carrera de licenciados en administración, para determinar beneficios y dificultades como herramienta de apoyo en el proceso de enseñanza - aprendizaje y evaluación de estudiantes universitarios.

Existen investigaciones previas que muestran información similar sobre el presente tema a investigar Uso de Smartphone como herramienta de apoyo en el proceso de enseñanza - aprendizaje y evaluación de estudiantes universitarios, beneficios $\mathrm{y}$ dificultades. como es el de González y Salcines (2015) El Smartphone en los procesos de enseñanza aprendizaje-evaluación en Educación superior. Percepción de docentes y estudiantes. Así mismo Cruz, Soberanes y Lule (2016) Análisis del smartphone como herramienta de apoyo en la formación académica de alumnos universitarios. Dichos estudios muestran a través de sus resultados que actualmente el uso del smartphone como herramienta de apoyo en la educación, está siendo muy relevante en la formación académica y de aprendizaje en el entorno universitario, además de señalar los beneficios y dificultades de su utilización en la educación superior.

\subsection{Justificación}


Considerando las desarrolladas habilidades y competencias que muestran los actuales estudiantes universitarios en el uso de la tecnología, y aplicados a la educación a distancia por la pandemia Covid 19, se requiere en los docentes la habilidad de aprender a usar y adaptar sus métodos de enseñanza a los requerimientos de los nuevos instrumentos de acceso y comunicación, como lo son los dispositivos móviles, además de explorar nuevas aplicaciones educativas que faciliten la formación académica del estudiante y mejoren su aprendizaje significativo.

\subsection{Objetivo}

Identificar el uso del smartphone en tiempos de pandemia en los estudiantes de séptimo semestre de la carrera de Licenciados en Administración, para determinar beneficios y dificultades como herramienta de apoyo en el proceso de enseñanza -aprendizaje y evaluación de estudiantes universitarios

\subsection{Hipótesis}

Ha: Los estudiantes universitarios utilizan el Smartphone como herramienta de apoyo en el proceso de enseñanza aprendizaje y evaluación.

Ha: El uso del Smartphone presenta beneficios y dificultades como herramienta de apoyo en el proceso de enseñanza aprendizaje y evaluación en los estudiantes universitarios.

Ho: Los estudiantes universitarios no utilizan el Smartphone como herramienta de apoyo en el proceso de enseñanza aprendizaje y evaluación.

Ho: El uso del Smartphone no presenta beneficios y dificultades como herramienta de apoyo en el proceso de enseñanza aprendizaje y evaluación en los estudiantes universitarios.

\section{MARCO TEÓRICO}

El uso del smartphone en tiempos de pandemia COVID-19 se ha incrementado notablemente por sus cualidades de funcionalidad, atendiendo a las diversas necesidades de sus usuarios en comunicación y acceso inmediato a la información, adaptándose a los requerimientos que día a día se presentan en los diferentes entornos en los que se desarrolla un individuo (laboral, social, personal y educación).

Con el paso de los años, la tecnología ha ido evolucionando de manera constante, los teléfonos inteligentes han transformado el estilo de vida de la sociedad; han llegado para quedarse entre todos y cada una de las personas. En 2017, según datos del INEGI, el $72.2 \%$ de la población de seis años o más utiliza el teléfono celular, 8 de cada 10 disponen de un smartphone, lo cual indica la posibilidad de conexión a Internet. Independientemente de edad, ingresos, género y el lugar de vida, el smartphone se ha vuelto una parte importante de la vida de los seres humanos. (p.1)

De manera que podemos precisar que: Smartphone es un teléfono celular con pantalla táctil y un robusto sistema operativo con el que los usuarios pueden conectarse a internet, instalar aplicaciones y llevar a cabo muchas de las actividades que se podrían realizar en una computadora. Aunque casi todos los celulares convencionales ya incluyen algún tipo de software (incluso los modelos más básicos, con libreta de direcciones o gestor de contactos), un teléfono inteligente tiene la capacidad de hacer mucho más. Las aplicaciones son pequeños programas creados específicamente para dispositivos móviles y permiten obtener una experiencia más adaptada y más fluida. Cuando se accede a través de un smartphone, por ejemplo, Facebook, fácilmente podríamos acceder desde nuestro smartphone utilizando el explorador, pero la experiencia no es para nada parecida a la que obtenemos al utilizar la aplicación. (Lozano,2020)

\subsection{El smartphone en las actividades academicas}

Por lo general, hoy en día la mayoría de los jovenes disponen de un telefono móvil ( smartphone ) pues para ellos las redes sociales son de gran interés. En el medio universitario se ha incrementado el uso de la comunicación 
virtual y disminuición de la comunicación presencial.

De acuerdo con Torres Díaz, Torres Carrión e Infante (2015), «el futuro del aprendizaje, desde una perspectiva técnica, está integrado por cuatro ejes que lo definen, sobre los que se articulan esfuerzos tecnológicos y metodológicos, como: la movilidad, interacción, inteligencia artificial y recursos basados en tecnología como la realidad aumentada y los juegos aplicados al aprendizaje». A nivel pedagógico, los dispositivos se constituyen en herramientas de interacción, pero se corre el riesgo de implementar fuentes de distracción y pérdida de la atención de los estudiantes. (Silva,2017, p.12)

\subsection{El Smartphone como apoyo en las actividades académicas en preparatoria, universidad y posgrado}

El uso de la tecnología ha venido a transformar la educación, en todos los niveles escolares, siendo indispensable el utilizar como herramienta de aprendizaje este dispositivo (smartphone) en la búsqueda y obtención de información; así como también para realizar tareas profesionales con una mejor presentación, además los docentes han implementado diversos métodos que ayudan a sus estudiantes a aprender conceptos y aplicar estrategias que motiven su aprendizaje.

Los estudiantes de nivel superior comparten la idea de que la posesión de un smartphone es beneficiosa; sin embargo, el uso de ellos se ha visto relacionado con un incremento de ansiedad. Ofrecen una experiencia única y permiten estar conectados con todo en cualquier momento y en cualquier lugar; pero también ha habido muchas denuncias contra ellos como: perder el tiempo, dejar los estudios inconclusos, evitar obligaciones, provocar actitudes desinteresadas, distracciones durante conducción, etc

\subsection{Uso y aplicación del Smartphone en el proceso de enseñanza-aprendizaje}

El aprendizaje móvil es, según Brazuelo y Gallego (2012), "Una modalidad educativa que, a través del uso de dispositivos móviles, facilita la construcción del conocimiento, la resolución de problemas y el desarrollo de habilidades".

Por otra parte, la Unesco (2013) define el uso de tecnología móvil para facilitar el aprendizaje en cualquier momento y lugar.

Se han traspasado las barreras para la educación especial, que involucran softwares, estrategias y procesos de aprendizaje para la inclusión. En su estudio, Sarwar y Soomro (2013) concluyen que el Smartphone facilita el acceso de la sociedad moderna a una enorme cantidad de recursos educativos y de aprendizaje. (Silva,2017, p.14)

Ahora sabemos y nos damos cuenta que el teléfono celular no solo es para la comunicación por llamada si no que nos ayuda en muchísimas tareas ya que es multifuncional.

De manera general, podemos manifestar que los teléfonos inteligentes son de gran uso entre la gran mayoría de los estudiantes y ofrecen capacidades asombrosas al contar con aplicaciones excelentes para mejorar el proceso de enseñanza y aprendizaje, pero al mismo tiempo, estas mismas aplicaciones podrían representar enormes distracciones y tener un impacto negativo en los estudiantes de manera que la forma en que se usan en los entornos de las aulas, debe pensarse y diseñarse cuidadosamente para lograr resultados positivos en todo momento.

Entre los jóvenes el uso de los smartphone es muy relevante, y para ellos son herramientas tecnológicas muy indispensables que les permiten crear, compartir y modificar información. Los Smartphone han venido a rediseñar el futuro educativo, como una herramienta de apoyo para el aprendizaje en la formación académica, aportando a la educación no solo movilidad sino también conectividad, al facilitar el manejo de información y transformarla en conocimiento. 
Es muy importante considerar lo que establecen Cataldi y Dominighini (2015), quienes argumentan que a los millennials se les pueden atribuir características generales, como: una niñez llena de actividades, una cultura de lo inmediato que ha marcado sus estilos vidas y de consumo. Son jóvenes que, nacidos en un contexto social con medios tecnológicos y de comunicación a su alcance, utilizan estos recursos en forma productiva y los consideran parte de la vida cotidiana. Les resulta vital estar conectados a través de los medios tecnológicos que no sólo son un mecanismo de comunicación sino también de socialización. (p.2)

Poseen una gran capacidad multitarea que significa una alternativa respecto del pensamiento lineal y estructurado, con una fuerte orientación a los fines, en relación a su desarrollo personal. Por lo cual los profesores, nacidos en generaciones previas, deben cambiar sus formas de enseñanza, haciendo esfuerzos para hacer sus asignaturas atractivas e incorporar a Internet y sus recursos como parte de sus formas de enseñanza. Pero los cambios, deben estar sustentados por las teorías que subyacen en las nuevas formas de aprendizaje.

\section{MÉTODO}

La presente investigación se considera de tipo cuantitativa, descriptiva y longitudinal, aplicada a una muestra de 169 estudiantes que cursan actualmente el séptimo semestre en el turno nocturno de la carrera de licenciados en administración, en una escuela de negocios del Noreste de México.

El cuestionario "Smartphone y Universidad. Visión del alumnado. SUOS" consta de 144 preguntas, distribuidas en tres bloques y nueve dimensiones teóricas, algunas de ellas subdimensionadas, tal y como se muestra en la tabla 1.

El cuestionario "Smartphone y Universidad. Visión del alumnado. Fue elaborado por Salcines Talledo Irina y González Fernández Natalia de la Universidad de Cantabria. Facultad de Educación.

Para facilitar la cumplimentación y recogida de datos, el cuestionario fue enviado a través del enlace https://forms.office.com/Pages/ResponsePage .aspx?id=EZDKymp73kSGHwlaLKiDtz6KZ oXNzvlIo9aCvteiqyNURjNRNzFJVU9TTTZ JMEpSVIVBWjQ2MUNWSC4u siendo el formato original el de la plataforma online: https://encuestas.unican.es/encuestas/index.ph p/779387/lang-es (Enlace de acceso al cuestionario)

Tabla 1 Estructura del cuestionario "Smartphone y Universidad. Visión del Alumnado"

$\begin{array}{ll}\text { Bloques } & \text { No. ítems }\end{array}$

BLOQUE 1: Datos Identificativos

BLOQUE 2: Preguntas generales sobre Smartphone

$\begin{array}{lll}\text { Dimensión 1: Conocimiento } & & 4 \\ \text { Dimensión 2: Uso } & \text { Lugar de uso } & 6 \\ & \text { Frecuencia de uso } & 8 \\ \text { Dimensión 3: Importancia } & & 5 \\ \begin{array}{l}\text { Dimensión 4: Introducción } \\ \text { pautada del Smartphone en } \\ \text { el proceso de E/A/E }\end{array} & \text { Beneficios } & 6 \\ \begin{array}{l}\text { Dimensión 5: Introducción } \\ \text { por iniciativa personal del }\end{array} & \text { Beneficios } & 5 \\ \begin{array}{l}\text { Smartphone en el proceso de } \\ \text { E/A/E }\end{array} & & 8 \\ & \text { Dificultades } & 6\end{array}$




$\begin{array}{llc}\text { Dimensión 6: Formación } & 6 \\ \begin{array}{l}\text { Dimensión7: } \\ \text { Conocimiento Aplicaciones }\end{array} & \text { Comunicación } & 5 \\ & \begin{array}{l}\text { Gestión y } \\ \text { Organización } \\ \text { Enseñanza/ } \\ \text { Aprendizaje/ } \\ \text { Evaluación }\end{array} & 7 \\ \text { Dimensión 8: } & \begin{array}{l}\text { Comunicación } \\ \text { Uso Aplicaciones }\end{array} & \mathbf{5} \\ & \begin{array}{l}\text { Gestión y } \\ \text { Organización }\end{array} & \mathbf{7} \\ & \begin{array}{l}\text { Enseñanza/ } \\ \text { Aprendizaje/ } \\ \text { Evaluación }\end{array} & \mathbf{1 4}\end{array}$

Nota. Recuperado de "Los Smartphones en la educación superior. Diseño y Validación de dos instrumentos de recogida de información sobre la visión del alumnado," de Salcines, I., \& González, N., 2015, REOP, 26 (3), p.101.Copyright 2015 de rip.

\section{RESULTADOS}

Para el análisis de los datos se llevó a cabo en primera instancia un análisis descriptivo correspondientes a las características de la muestra, que estaban incluidas en el bloque 1 del cuestionario. Dentro de las cuales se encuentran: sexo, edad, semestre, y disposición de smartphone, y el tiempo que utiliza durante el día el smartphone.

El tamaño de la muestra validado fue de 169 cuestionarios efectivos, lo cual se considera un número adecuado según Gaitán y Piñuel (1998) el análisis de fiabilidad para el cuestionario se determinó mediante el cálculo del alfa de Cronbach.
Para el análisis de las dimensiones se utilizó el análisis factorial para el bloque 2 Para lo cual se llevaron a cabo las pruebas de KMO y Bartlett en cada uno de los análisis factoriales realizados.

\subsection{Características de la muestra}

Mediante el análisis descriptivo se ha determinado las características más relevantes de la muestra, esta información fue recabada por los ítems del 1 al 7 dentro del bloque 1 del cuestionario. En la tabla 2 se observa la clasificación por sexo de la muestra, siendo las mujeres la mayoría con un $54 \%$ de representación en la muestra.

Tabla 2. Clasificación por sexo.

\begin{tabular}{ll}
\hline Sexo & $\%$ \\
\hline Hombre & $46 \%$ \\
Mujer & $54 \%$ \\
\hline
\end{tabular}

Fuente: Elaboración Propia

La tabla 3 nos ofrece la observación de la composición por edad de la muestra, siendo la de mayor porcentaje los alumnos con edad entre 20 y 25 años. Este dato es consistente con el semestre, ya que la mayoría de los alumnos de la muestra cursa un semestre avanzado. En la tabla 4 podemos observar que un $96 \%$ de los alumnos de la muestra, cursa el séptimo semestre.

Tabla 3. Clasificación por edad.

Edad $\%$




\begin{tabular}{ll} 
De 20 a 25 años & $94 \%$ \\
Más de 25 años & $4 \%$ \\
Menor de 20 años & $2 \%$ \\
\hline
\end{tabular}

Fuente: Elaboración Propia

Tabla 4. Clasificación por semestre.

\begin{tabular}{cc}
\hline Semestre en curso & $\%$ \\
\hline 3 & $1 \%$ \\
7 & $96 \%$ \\
8 & $3 \%$ \\
\hline \multicolumn{2}{c}{ Fuente: Elaboración Propia }
\end{tabular}

La mayoría de los alumnos de la muestra un $98 \%$ de los alumnos tiene un teléfono tienen un smartphone, esto lo podemos inteligente o Smartphone. observar en la tabla 5, donde se muestra que

Tabla 5. Disponibilidad de Smartphone.

\begin{tabular}{lc}
\hline \multicolumn{2}{l}{ Dispone de un Smartphone } \\
\hline No & $2 \%$ \\
$\mathrm{Si}$ & $98 \%$ \\
\hline
\end{tabular}

Fuente: Elaboración Propia

Conocer cuánto tiempo diario utilizan el smartphone para fines académicos, es fundamental para la contextualización del análisis, en la tabla 6 de muestra las categorías por tiempo de uso del dispositivo, y se puede observar que al menos un 64\% utiliza durante el día, más de tres horas el Smartphone.

Tabla 6. Tiempo de uso del Smartphone para fines académicos.

\begin{tabular}{ll}
\hline Tiempo diario de uso & $5 \%$ \\
\hline 0 horas & $9 \%$ \\
En torno a 1 hora diaria. & $18 \%$ \\
En torno a 2 horas diarias. & $64 \%$ \\
En torno a 3 o más horas diarias & $2 \%$ \\
Menos de 1 hora diaria. & \\
\hline \multicolumn{2}{c}{ Fuente: Elaboración Propia }
\end{tabular}

\subsection{Análisis y Discusión}

El bloque 1 del cuestionario nos fue de gran utilidad para conocer las características más 
relevantes de la muestra, para el análisis con respecto a la visión del alumnado sobre el Smartphone y la universidad.

El bloque 2 del cuestionario se adentra en la relación con las actividades académicas mediante el uso del Smartphone, para esto se llevará a cabo un análisis factorial para los ítems del 8 al 18 que, están dentro del bloque 2 , excluyendo el ítem 12 , ya que es un ítem de clasificación de tipo cadena.
Para determinar la fiabilidad del cuestionario en relación a los ítems que corresponden al bloque 2, se determinó el alfa de Cronbach. En la tabla 7 se presenta el cálculo de alfa de Cronbach $=0.917$ para el bloque 2 del cuestionario de Smartphone y universidad, que está dentro del límite establecido para determinar una fiabilidad del bloque 2 .

Tabla 7. Alfa de Cronbach para la fiabilidad del bloque 2 del cuestionario.

\begin{tabular}{lll}
\hline $\begin{array}{l}\text { Medida de adecuación } \\
\text { muestral de Kaiser-Meyer- } \\
\text { Olkin. }\end{array}$ & 0.826 \\
\hline $\begin{array}{l}\text { Prueba de } \\
\text { esfericidad }\end{array}$ & Chi-cuadrado & \\
de Bartlett & gl & 6659.137 \\
& Sig. & 1431 \\
& 0.000 \\
\hline
\end{tabular}

\begin{tabular}{cc}
\hline \multicolumn{2}{c}{ Estadísticos de fiabilidad } \\
\hline Alfa de \\
Cronbach & N de elementos \\
\hline 0.917 & 54
\end{tabular}

Fuente: Elaboración Propia

Para asegurar la calidad del análisis y viabilidad, se determinó el KMO y la prueba de esfericidad de Bartlett para el bloque 2 del cuestionario. La tabla 8, nos muestra los resultados de las pruebas donde el $K M O=$
0.826 y el índice de significancia (Sig.) en este caso es de 0.000 , con lo cual se validan los datos para determinar los factores más relevantes.

Tabla 8. KMO y Prueba de Bartlett para bloque 2 del cuestionario. 
Fuente: Elaboración Propia

Para el bloque 2 que corresponden los ítems del 8 al 18, excluyendo el ítem 12, se calculó un análisis factorial para encontrar los componentes o dimensiones más relevantes.

En la tabla 9 nos muestra los componentes más relevantes para el bloque 2 del cuestionario, donde se observa que esté compuesto por 5 factores o componentes. El primer factor representa el $20.23 \%$ de la varianza, y en conjunto los 5 factores acumulan un $54.90 \%$ de varianza.

Tabla 9. Factores o componentes más relevantes, en el bloque 2 del cuestionario.

\begin{tabular}{llll}
\hline \multirow{4}{*}{ Componente } & \multicolumn{3}{l}{ Auto valores iniciales } \\
\cline { 2 - 4 } & Total & $\begin{array}{l}\text { \% de la } \\
\text { varianza }\end{array}$ & acumulado \\
\cline { 2 - 4 } & & & \\
\hline 1 & 10.925 & 20.232 & 20.232 \\
2 & 7.744 & 14.340 & 34.572 \\
3 & 5.029 & 9.312 & 43.884 \\
4 & 3.505 & 6.491 & 50.375 \\
5 & 2.446 & 4.529 & 54.904 \\
\hline
\end{tabular}

Fuente: Elaboración Propia

El análisis factorial del bloque 2 que dentro de los cuales se agrupan distintos comprende la relación académica con el ítems. smartphone, nos dio por resultado 5 factores,

Tabla 10. Factores relevantes en la relación del Smartphone con las actividades académicas.

\begin{tabular}{|c|c|c|c|c|c|c|c|c|c|}
\hline \multicolumn{2}{|c|}{$\begin{array}{l}\text { Factor 1: } \\
\text { Utilidad y } \\
\text { acceso de } \\
\text { informació } \\
\text { n }\end{array}$} & \multicolumn{2}{|c|}{$\begin{array}{l}\text { Factor 2: } \\
\text { Auxiliar en el } \\
\text { aprendizaje }\end{array}$} & \multicolumn{2}{|c|}{$\begin{array}{l}\text { Factor 3: } \\
\text { Desventajas } \\
\text { del uso del } \\
\text { Smartphone }\end{array}$} & \multicolumn{2}{|c|}{$\begin{array}{l}\text { Factor 4: } \\
\text { Ventajas del } \\
\text { uso del } \\
\text { Smartphone }\end{array}$} & \multicolumn{2}{|c|}{$\begin{array}{l}\text { Factor 5: } \\
\text { Compleme } \\
\text { nto para el } \\
\text { aprendizaje }\end{array}$} \\
\hline $15 \mathrm{e}$ & 0.806 & $10 \mathrm{c}$ & 0.803 & $14 \mathrm{c}$ & 0.751 & 13a) & 0.804 & $9 \mathrm{~d}$ & 0.797 \\
\hline $15 \mathrm{f}$ & 0.801 & $10 \mathrm{a}$ & 0.757 & $14 \mathrm{~b}$ & 0.735 & $13 \mathrm{~d}$ & 0.7914 & $9 f$ & 0.716 \\
\hline $15 b$ & 0.766 & $10 \mathrm{f}$ & 0.755 & $16 f$ & 0.707 & $13 \mathrm{f}$ & 0.7873 & $9 a$ & 0.676 \\
\hline $15 \mathrm{a}$ & 0.751 & $10 \mathrm{~d}$ & 0.746 & $16 \mathrm{c}$ & 0.685 & $13 \mathrm{c}$ & 0.7861 & $9 \mathrm{e}$ & 0.650 \\
\hline $15 \mathrm{c}$ & 0.748 & $10 \mathrm{c}$ & 0.735 & $16 \mathrm{e}$ & 0.65 & $13 b$ & 0.7559 & $9 \mathrm{c}$ & 0.649 \\
\hline $15 f$ & 0.745 & $10 \mathrm{~b}$ & 0.721 & $14 d$ & 0.639 & $13 e$ & 0.7536 & $9 b$ & 0.591 \\
\hline $15 \mathrm{~g}$ & 0.716 & $10 \mathrm{e}$ & 0.668 & $14 \mathrm{e}$ & 0.631 & & & & \\
\hline $15 d$ & 0.707 & $10 \mathrm{~g}$ & 0.552 & $14 \mathrm{a}$ & 0.615 & & & & \\
\hline & & & & $16 b$ & 0.549 & & & & \\
\hline
\end{tabular}


En la tabla 10 se puede observar los factores más relevantes y los ítems junto con sus respectivos índices, los cuales son derivados del cálculo de la matriz rotada del análisis factorial del bloque 2 .

El análisis y resultados nos indican que los alumnos utilizan más su smartphone dentro de sus actividades académicas, por la seguridad y comodidad de utilizar su smartphone, para el acceso y búsqueda de información.

Dentro de las actividades que más le encuentran utilidad, son la realización de actividades de aprendizaje, el acceso a exposiciones magistrales y el desarrollo del portafolio de las unidades de aprendizaje. Pero también se muestran preocupados por las desventajas del uso del Smartphone, ya que pude llegar a interrumpirlos o disminuir la atención en las sesiones. Dentro de las ventajas que valoran, están un aumento de motivación y creatividad.

Además, valoraron al Smartphone como un complemento, para el acceso a bibliotecas, para la comunicación entre los compañeros, así como la gestión y la organización de sus actividades académicas.

\section{CONCLUSIÓN}

El análisis de los resultados nos indica que la gran mayoría de los alumnos, esto es un $98 \%$ (tabla 5) tienen acceso o dispone de un Smartphone. Y hasta un $64 \%$ de los alumnos pasa más de 3 horas en el smartphone para actividades académicas (tabla 6). Con lo cual se puede inferir que hay un uso masivo y prolongado del smartphone para las actividades académicas.

Las pruebas de fiabilidad y viabilidad del bloque 2 fueron satisfactorias, por lo que, el análisis factorial y sus resultados de los ítems del bloque 2, nos ofrecen una buena inferencia sobre estudio de la relación del Smartphone con las actividades académicas de los alumnos en tiempos de pandemia.

El análisis factorial nos reveló cinco factores o componentes relevantes, que en total acumulan el 54.90\% de la varianza (Tabla 9). Siendo el factor principal la utilidad y acceso de información que les provee el Smartphone para las actividades académicas (Tabla 10). Esto es muy relevante ya que debido a la actual pandemia del COVID 19, las clases presenciales se suspendieron desde finales del mes de marzo del año 2020, y así continuaran por lo menos el semestre Febrero - Junio del 2021, según las autoridades educativas universitarias. Ante esta situación se empezaron a dar las clases mediante plataformas online. Por lo que, se puede inferir que los alumnos han visto en su Smartphone una herramienta de gran utilidad para realizar sus actividades académicas.

Además, el análisis de los resultados, indican que los alumnos consideran al dispositivo como un auxiliar en el aprendizaje, aunque también reconocieron las distintas desventajas del Smartphone, tales como la interrupción en las sesiones o clases, o la disminución de atención.

Pero también reconocieron como ventajas del uso del Smartphone el aumento de la motivación y la creatividad, además de considerar al smartphone como un complemento para la gestión y organización de sus actividades académicas, tanto para la utilización de agendas, acceso a bibliotecas y como un canal de comunicación entre compañeros.

Por lo que, se puede concluir que ante la actual pandemia del COVID 19, el uso del Smartphone es de un alto porcentaje y prologando durante el día, debido al nuevo contexto educativo de tomar las clases mediante plataformas online, los alumnos se han apoyado en el uso del Smartphone para llevar a cabo sus actividades académicas.

Se concluye también que los alumnos muestran como más sobresalientes algunas desventajas tales como la interrupción en las sesiones o en la atención, ya que es frecuente recibir notificaciones del uso de las redes sociales lo cual los distrae e incluso los involucra durante largos lapsos de tiempo, sin que se den cuenta; aunque también se mostró 
una importancia por las ventajas, tales como el aumento de la motivación y creatividad para realizar sus actividades al utilizar diversas herramientas a través de su smartphone.

También es necesario mencionar que estos dispositivos (smartphone) al igual que los aparatos electrónicos son dependientes de la electricidad y el acceso a internet, por lo cual hay que tener un equilibrio en su dependencia.
Otra conclusión que podemos establecer como significativa en el uso del smartphone en la educación universitaria es que, por motivos de la pandemia, en casa los padres hacen home office, además de los hermanos que estudian en otros niveles educativos, lo cual obliga a los universitarios a tomar sus clases en diversos medios digitales que no son computadoras de escritorio o laptop. 


\section{REFERENCIAS}

Cataldi, Z. \& Dominighini, C. (2015). La generación millennials y la educación superior. Los retos de un nuevo paradigma. Revista de informática educativa y medios audiovisuales. 12(19), 14-21. http://www.codajic.org/sites/www.codajic.org/files/La\%20generaci\%C3\%B3n\%20millenni al\%20y\%20la\%20educaci\%C3\%B3n\%20superior..pdf

Cruz, A., Soberanes, A. \& Lule, A. (2016). Análisis del Smartphone como herramienta de apoyo en la formación académica de alumnos universitarios. Pistas educativas. 38(122),135-155.

Obtenido http://www.itcelaya.edu.mx/ojs/index.php/pistas/article/viewFile/695/621

Figueroa, C. (2016). El uso del smartphone como herramienta para la búsqueda de información en los estudiantes de pregrado de educaión de una Universidad de Lima metropolitana. Scielo 25(49),29-44. Obtenido de http:// www.scielo.org.pe/pdf/educ/v25n49/a02v25n49.pdf

González,N.,\& Salcines, I.(2015). El Smartphone en los procesos de enseñanza-aprendizajeevaluación en Educación Superior. Percepciones de docentes y estudiantes. RELIEVE. 21(2), 1-20. Obtenido de https://www.redalyc.org/pdf/916/91643847006.pdf

INEGI. (2018). En México 71.3 millones de usuarios de internet y 17.4 millones de hogares con conexión a este servicio: Endutih 2017.Comunicado de Prensa. Encuesta Nacional sobre Disponibilidad. 20-02-2018, de INEGI Sitio web: https://www.inegi.org.mx/contenidos/saladeprensa/boletines/2018/OtrTemEcon/ENDUTIH 2018_02.pdf

Lozano, M. (17 de febrero, 2020). whistle out. Obtenido de https://www.whistleout.com.mx/CellPhones/Guides/que-es-un-smartphone

Organista, J., Salas, L. \& Lavinge, G. (2013). El teléfono inteligente (Smartphone) como herramienta pedagógica. Apertura 5 (1), 6-19. Obtenido de https://www.redalyc.org/pdf/688/68830443002.pdf

Salcines, I. \& González, N. (2015). Los smartphones en educación superior. Diseño y Validación de dos instrumentos de recogida de información sobre la visión del alumnado. REOP 26(3), 96120. Obtenido de https://www.redalyc.org/pdf/3382/338245392006.pdf

Silva, A.C. \& Martínez, D.G. (2017). Influencia del Smartphone en los procesos de aprendizaje y enseñanza. Suma de Negocios. 8(2017), 11-18. Obtenido de https://www.sciencedirect.com/science/article/pii/S2215910X17300010\#bib0215 